\title{
Long-term trends and decadal variability of upper mesosphere/lower thermosphere gravity waves at midlatitudes
}

\author{
Ch. Jacobi \\ Institut für Meteorologie, Universität Leipzig, Stephanstr. 3, 04104 Leipzig
}

\section{Summary}

Mesosphere/lower thermosphere (MLT) winds over Germany as measured with a lowfrequency spaced receiver system at Collm 1984-2007 have been analysed with respect to variations at the time scales of gravity waves. Background winds are also registered to analyse gravity-mean flow interactions at decadal and interdecadal time scales. In both winter and summer an increasing mesospheric zonal wind jet with time is registered, which is connected with increasing gravity wave variances. At greater altitudes in summer, the mean wind jet trend reverses, and negative trends of gravity wave variances are found. This connection between gravity waves and mean wind is also observed on a quasi-decadal scale: during solar maximum stronger mesospheric zonal wind jets as well as larger gravity wave amplitudes are observed. This results in a solar cycle modulation of gravity waves with larger amplitudes during solar maximum. The connection between gravity waves and mean zonal wind may be explained by wave filtering within linear wave theory, such that stronger mesospheric zonal winds are connected with larger gravity wave amplitudes.

\section{Zusammenfassung}

Horizontalwinde, gemessen 1984-2007 mit einem Langwellen-Windprofilersystem in Collm wurden im Hinblick auf Langzeitvariationen kurzperiodischer Schwankungen, die als Signatur von Schwerewellen interpretiert werden können, ausgewertet. Der Grundwind wurde ebenfalls bestimmt, um Welle-Grundstrom-Wechselwirkungen auf der dekadischen und interdekadischen Zeitskala zu untersuchen. Sowohl im Sommer als auch im Winter wurde ein mit der Zeit zunehmender mesosphärischer Jet beobachtet, der mit zunehmenden Schwerewellenamplituden verbunden ist. In größeren Höhen dreht sich der Trend im Sommer um, verbunden mit negativem Trend der Schwerewellenaktivität. Dieser Zusammenhang lässt sich durch Schwerwellenfilterung im Rahmen linearer Theorie erklären. Der Zusammenhang zwischen Grundstrom und Schwerewellenaktivität ist auch auf der dekadischen Zeitskala zu sehen: im solaren Maximum sind die mesosphärischen Jets stärker und die Schwerewellenamplituden größer.

\section{Introduction}

Gravity waves (GWs) play a crucial role in maintaining the dynamics of the mesosphere/lower thermosphere (MLT) region. Their sources are mainly located in the troposphere, and they transport energy and momentum to the middle atmosphere, thus leading to a coupling between atmospheric layers. Changes of GW parameters and 
amplitudes are thus connected with changes in atmospheric coupling, and the analysis of long-term trends of GW may give insight into changes of the atmosphere as a whole. Acceleration of the mean flow through GWs mainly occurs in the mesosphere/lower thermosphere (MLT). This region is characterised by the wind reversal, i.e. the change of the mesospheric summer/winter easterly/westerly jets to the lower thermosphere westerly/easterly jets through GW momentum deposition.

The MLT region is accessible to radar wind measurements, although standard methods only deliver limited temporal and thus spectral resolution of GW. On the other hand, however, radar measurements are cost effective, reliable, and independent of weather and thus may provide long-term datasets of background prevailing wind and $\mathrm{GW}$ information. Consequently, some effort has been undertaken to analyse $\mathrm{GW}$ changes in the MLT also in connection with background wind (Jacobi et al., 2006; Hoffmann et al., 2011). GWs are filtered in the mesosphere through the zonal wind jets. Eastward/westward travelling GWs usually encounter critical lines in the winter/summer mesosphere, where the phase speed equals the wind speed. Therefore, essentially westward/eastward travelling GWs remain in the upper mesosphere. According to linear theory, in the case of wave saturation the GW amplitudes equal the intrinsic phase speed, so that in summer stronger mesospheric easterlies should be connected with larger GW amplitudes, while in winter stronger mesospheric westerlies lead to larger GW amplitudes.

Jacobi et al. (2006) has found a positive correlation of solar activity and GW proxies derived from Collm wind measurements. They attributed this correlation to the possible effect of a solar cycle variation of the mesospheric jet, which is stronger during solar maximum both in winter and in summer. Hoffmann et al. (2011), analysing 22 years of medium frequency radar wind data over Juliusruh, Germany, showed a longterm increase of the mesospheric wind jet and consequently an increase of GW kinetic energy in summer. Both of these findings can be explained by linear theory.

Here, a 24 year dataset of gravity wave proxies is analysed with respect to long-term changes as well as decadal variability, the latter mainly driven by the 11-year solar cycle. The dataset represents an update of the one used by Jacobi et al. (2006). In the following section 2 the method of GW proxy analysis is briefly described. Section 3 presents results of long-term mean prevailing winds and gravity wave proxies. In section 4, analysis of trends and solar cycle dependence is performed using linear leastsquares fitting. In section 4 the results are discussed in the light of linear GW theory. Section 6 concludes the paper.

\section{LF wind measurements and gravity wave proxy analysis}

From 1959-2007, MLT winds have been measured at Collm, Germany, using the spaced receiver method in the low-frequency (LF) range at oblique incidence. Commercial transmitters have been used, one of them located at Zehlendorf near Berlin (frequency $177 \mathrm{kHz}$ ), at a distance to Collm of about $165 \mathrm{~km}$. Thus, the reflection point of the sky wave registered at Collm is located at $52.1^{\circ} \mathrm{N}, 13.2^{\circ} \mathrm{E}$. Horizontal 
winds have been analysed using the similar fade method at 3 receivers at $300 \mathrm{~m}$ distance. The applied method makes use of the fact that corresponding maxima or minima of similar fadings of the sky wave are registered at the different receivers with a time delay with respect to each other, which is proportional to the drift velocity of electron density fluctuation patterns at the LF reflection height near $90 \mathrm{~km}$. The method has been described in detail, e.g., by Schminder and Kürschner (1995), and references therein.

The reflection height has been measured from late 1982 to 2007 on $177 \mathrm{kHz}$ through comparison of the phase of the ground wave and sky wave on a side-band frequency near $1.8 \mathrm{kHz}$ (Kürschner et al., 1987). Since the group velocity of radio waves in the lower ionosphere is lower than the speed of light, the resulting altitudes represent virtual heights, and these exceed the real heights by several kilometres. A correction, based on the comparison of semidiurnal tidal phase positions at corresponding heights using meteor radar (Jacobi, 2011) has been applied. Monthly median reflection heights range between 80 and $95 \mathrm{~km}$ (Figure 1).

The reflection heights change in a regular manner in the course of one day, since they broadly represent the altitude of a fixed electron density, which equals the critical frequency at oblique incidence. In addition, above all in summer regular data gaps appear during daylight hours (Figure 1, right panel). Therefore, estimates of daily height profiles of winds are not available. Monthly prevailing winds have thus been calculated from one month of half-hourly mean zonal and meridional winds and reflection heights. A least-squares fitting of the prevailing wind and the semidiurnal tide has been applied to these data. There, a second order height-dependence of the regression coefficient has been assumed. The method has been described, e.g., in Schminder and Kürschner (1988) and Jacobi (2011). Right-hand circular polarization of the horizontal tidal components has been assumed (Kürschner, 1991).
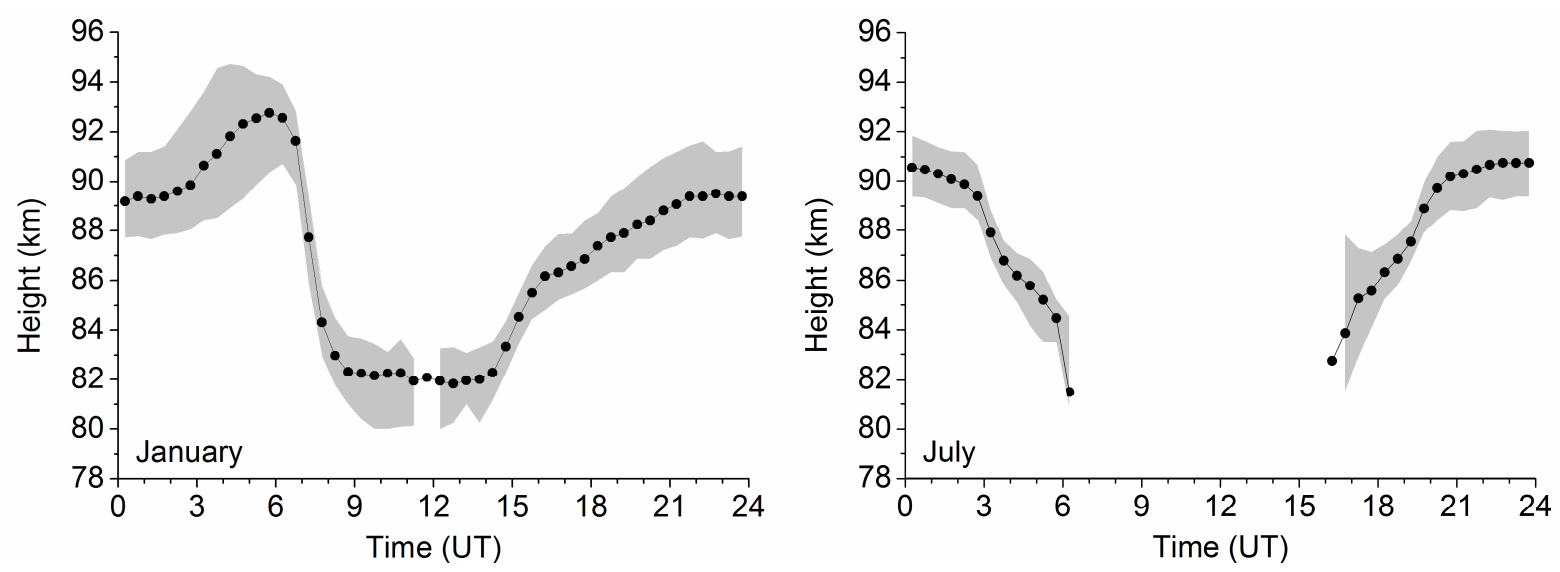

Fig. 1: 1983-2007 median reflection heights in January (left panel) and July (right panel). Upper and lower quartiles are indicated by the shaded area. 
Proxies of gravity wave variance in the period range 0.7-3 h (Gavrilov et al., 2001) have been calculated from the squared differences of subsequent half-hourly mean zonal or meridional winds, provided that the height difference of these means does not exceed $1 \mathrm{~km}$. Note that this means that GW proxies at different altitudes preferably refer to different times of the day (see Figure 1). The monthly mean variances have been calculated using data from one month of data within $7 \mathrm{~km}$ vertical windows each. The procedure is described in Gavrilov et al. (2001) and Jacobi et al. (2006), but there $10 \mathrm{~km}$ height windows and virtual heights have been used.

\section{Long-term mean prevailing winds and gravity wave proxies}

Long-term mean prevailing winds are presented in Figure $2 \mathrm{a}$ for the zonal and in Figure $2 \mathrm{~b}$ for the meridional component. The results are similar to those that have been presented, e.g., by Jacobi (2011), but with a different time interval under consideration and the height correction applied. Note that the height correction proposed by Jacobi (2011) relies on winter tidal phases only, since during summer the semidiurnal tidal wind gradient is small and thus the respective phases cannot be attributed to a defined altitude. This means that there is an additional uncertainty in the heights attributed to the summer winds.

The zonal prevailing winds show the upward extension of the mesospheric westerly and easterly jets in winter and summer, respectively. The zonal winds are decreasing with altitude, and eventually reversing, however, on a long-term average the zero wind line in winter lies above the region covered by the measurements. The meridional wind (Figure 2b) is mainly southward (negative), but northward in winter below about 83 $\mathrm{km}$. These jets are connected with gravity wave forcing of the zonal mean wind and residual circulation. The long-term means presented in Figure 2 qualitatively agree with results from other measurements (e.g., Hoffmann et al., 2011), but the amplitudes are biased towards smaller values compared with Doppler methods (e.g. Jacobi et al., 2009).

Long-term mean GW variances are presented in Figure 3. Generally, there are maximum amplitudes found in the summer upper mesosphere, and decreasing amplitudes with height. In the upper height gates, there is a tendency for increasing zonal amplitudes in winter. Comparing Figure 2 and Figure 3a, there is a tendency for (i) the southward meridional prevailing wind to maximise in the region of maximum zonal wind shear and (ii) the summer zonal GW variance maximising at regions of maximum zonal prevailing easterly (negative) wind. This would be consistent with linear GW theory, which predicts that, provided the GW are saturated, the GW amplitudes are proportional to the intrinsic phase speed and thus decrease with altitude when the zonal winds decrease or reverse. 
(a)

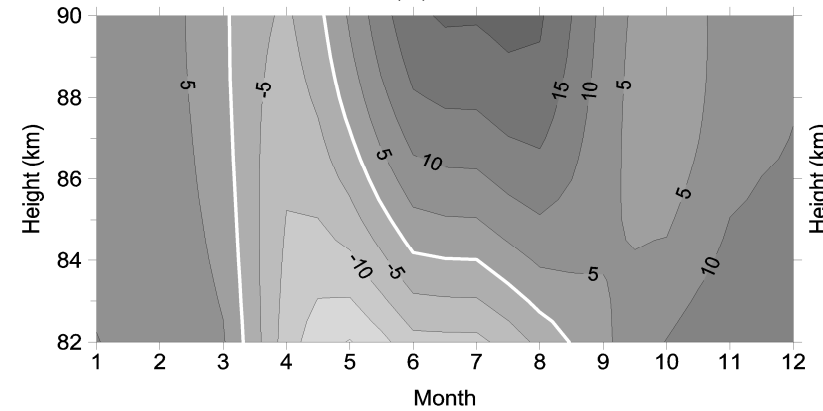

(b)

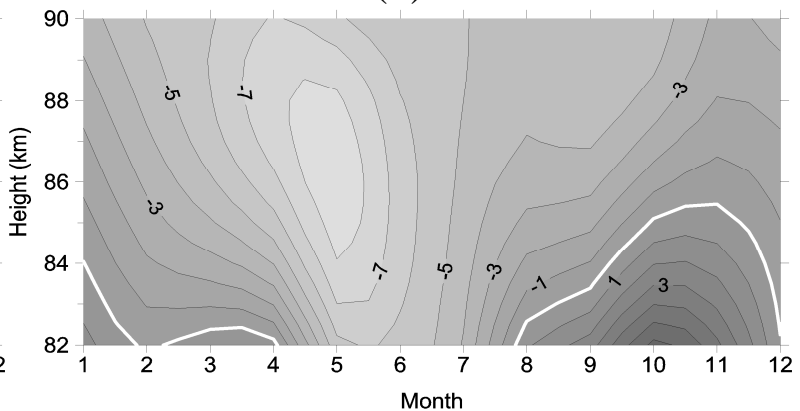

Fig. 2: Height-time cross-sections of the zonal ( $a$ ) and meridional $(b)$ prevailing wind over Collm. The data are 1984-2006 means based on regression analyses with quadratic height dependence of the coefficients and right-hand circularly polarized tidal components assumed.

(a)

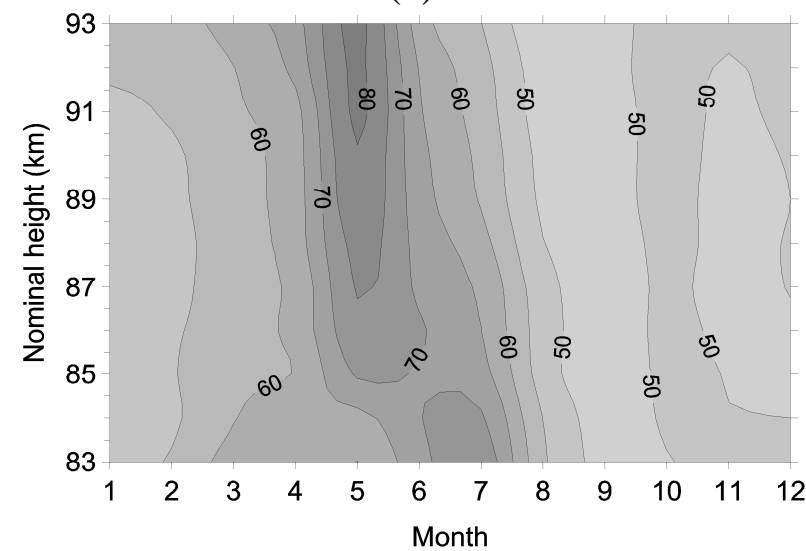

(b)

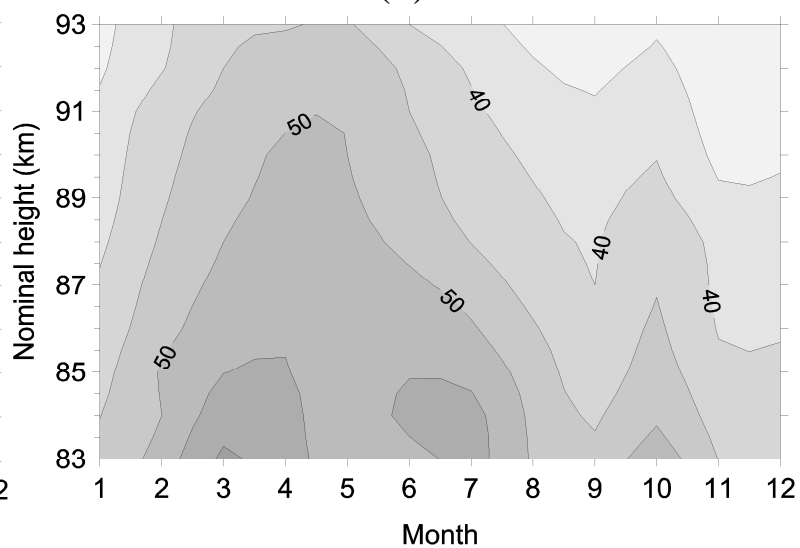

Fig. 3: 10/1983-9/2007 mean zonal (a) and meridional (b) variances over Collm, calculated from half-hourly wind differences (see Jacobi et al., 2006).

\section{Long-term trend and solar cycle analysis}

Jacobi et al. (2006) have already pointed out that there is an apparent solar cycle effect visible in both zonal and meridional variances over Collm. Regarding long-term changes, Hoffmann et al. (2011) presented long-term (22 years) trends of GW variances over Juliusruh, Germany, and found that summer GW variances have increased during that time interval, connected with an increase of the mesospheric wind jet. Therefore, here the GW variance time series are analysed with respect to a possible long-term change and a solar cycle by:

$$
\sigma^{2}=a+b \cdot y r+c \cdot F 10.7
$$

with $\sigma^{2}$ as the sum of the zonal and meridional variance and $F 10.7$ as the $10.7 \mathrm{~cm}$ radio flux given in solar flux units ( $\mathrm{sfu}, 1 \mathrm{sfu}=10^{-22} \mathrm{Wm}^{-2} \mathrm{~Hz}^{-1}$ ). The results, i.e. the coefficients $b$ and $c$, are presented in Figure 4. For comparison, the same kind of analysis has been applied to the zonal prevailing winds $v_{z o n}$, and the resulting regression coefficients are presented in Figure 5. 
(a)

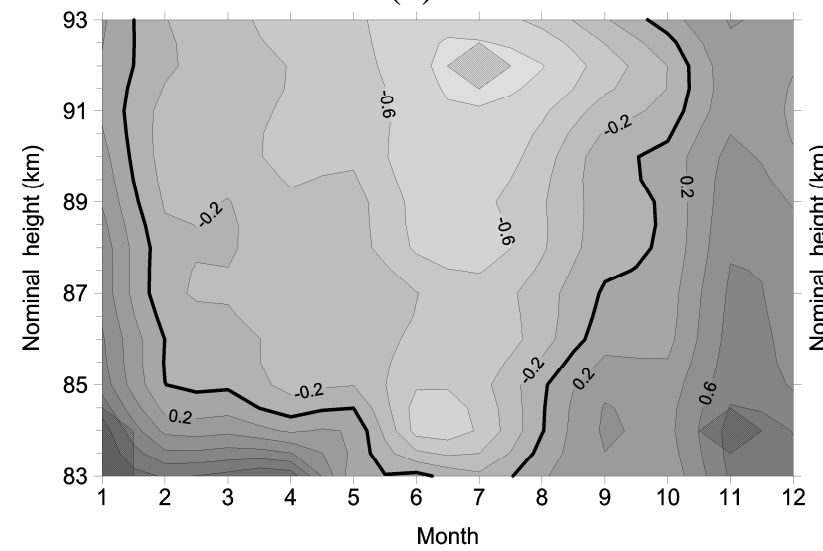

(b)

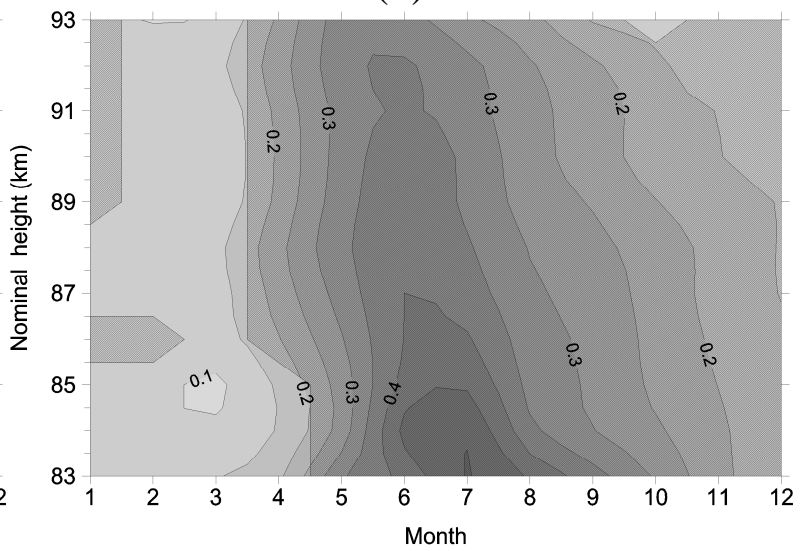

Fig. 4: Linear trend $b\left(m^{2} s^{-2} y r^{-1}\right)(a)$ and solar effect $c\left(m^{2} s^{-2} s f u^{-1}\right)(b)$ of $G W$ variance according to a regression analysis $\sigma^{2}=a+b \cdot y r+c \cdot F 10.7$ based on 1984-2007 3monthly means over Collm. Significant values at the $95 \%$ level according to a $t$-test are hatched.

(a)

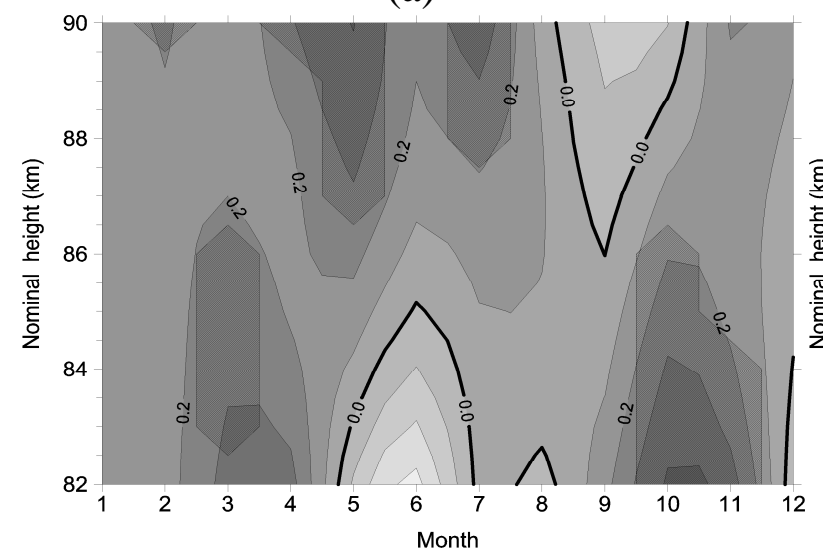

(b)

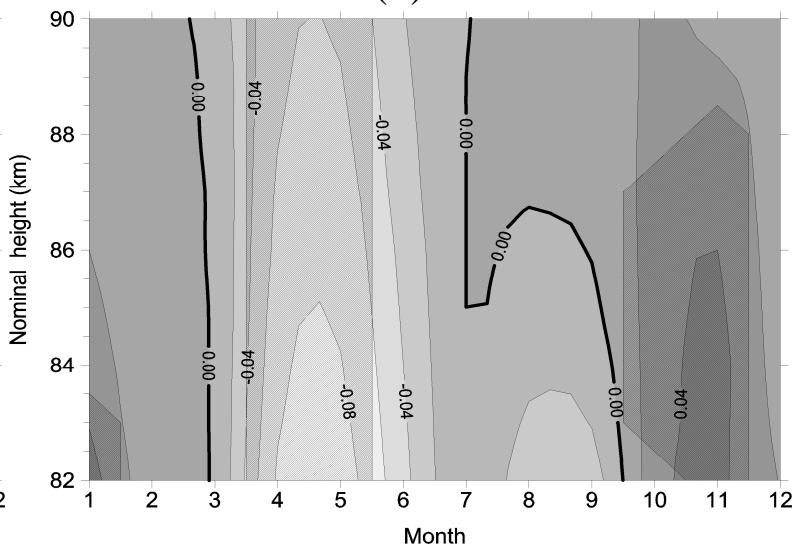

Fig. 5: Linear trend $b\left(m s^{-1} y r^{-1}\right)(a)$ and solar effect $c\left(m s^{-1} s f u^{-1}\right)(b)$ of the zonal prevailing wind according to a regression analysis $v_{z o n}=a+b \cdot y r+c \cdot F 10.7$ based on 1984-2007 3-monthly means over Collm. Significant values at the 95\% level according to a t-test are hatched.

One may see from Figures $4 \mathrm{~b}$ and $5 \mathrm{~b}$ that the strongest solar cycle signal of the zonal prevailing wind is found in late spring/early summer and early winter. Analysed longterm trends are weaker and mostly insignificant; however, again the strongest signal is seen in late autumn/early winter. Therefore, to illustrate the long-term variability of the mean wind and GW parameters, in Figures 6 and 7 time series of 3-monthly mean $\sigma^{2}$ and $v_{z o n}$ are presented for May-July and October-December means at different altitudes. Linear trends, although not always being significant (see Figures 4 and 5) are added. 
(a)

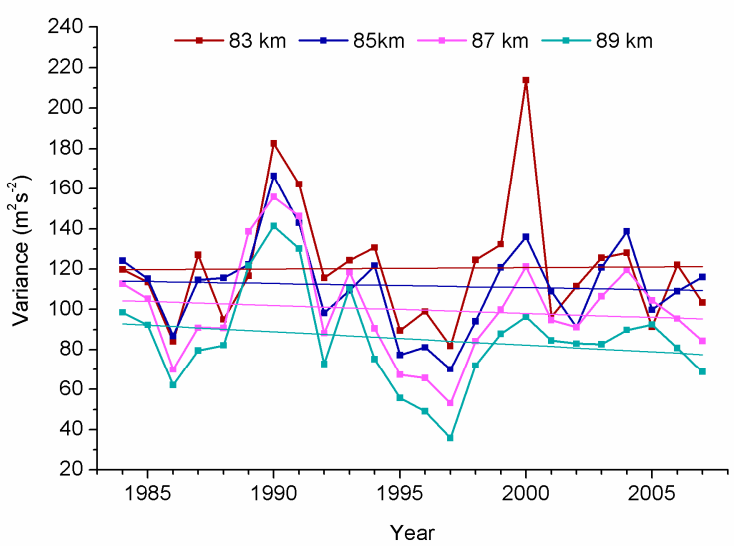

(b)

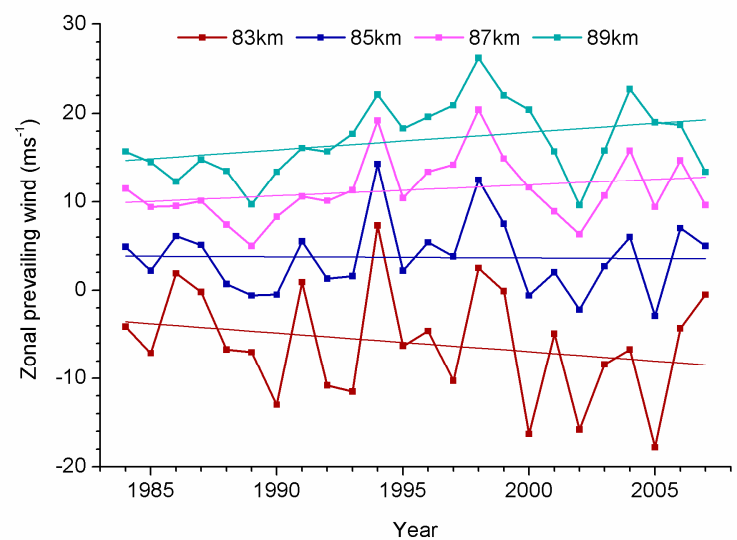

Fig. 6: May-July mean time series of variance $\sigma^{2}(a)$ and zonal prevailing wind $v_{z o n}(b)$ over Collm. Variances at 85/87/89 km have been shifted by $-10 /-20 /-30 \mathrm{~m}^{2} \mathrm{~s}^{-2}$.

(a)

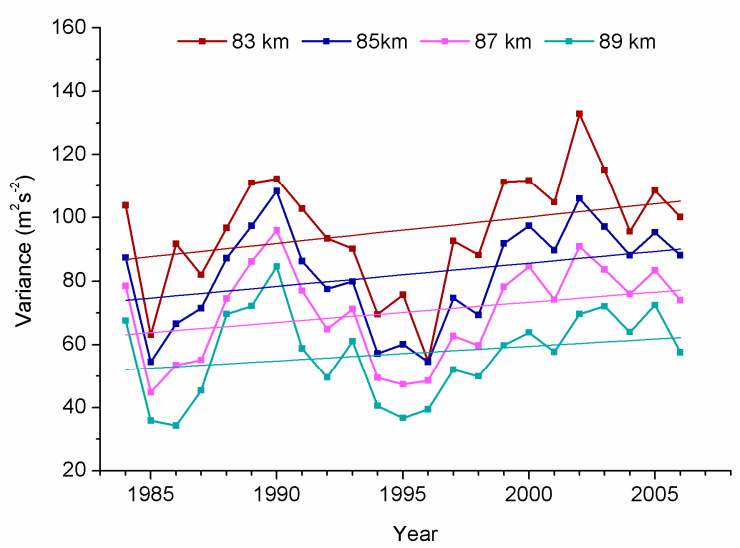

(b)

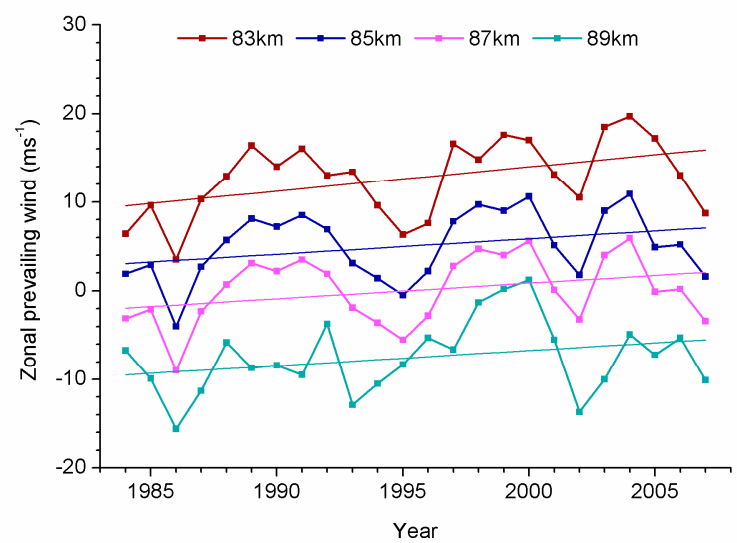

Fig. 7: October-December mean time series of variance (a) and zonal prevailing wind (b) over Collm. Variances at $85 / 87 / 89 \mathrm{~km}$ altitudes have been shifted by $-10 /-20 /-30 m^{2} s^{-2}$. Mean winds at 85/87/89 $\mathrm{km}$ have been shifted by -5/-10/-15 $\mathrm{ms}^{-1}$.

\section{Discussion}

Hoffmann et al. (2011) have presented negative summer prevailing wind trends, which indicate that the mesospheric summer easterly wind jet becomes stronger with time. This trend decreases with altitude and reverses in the lower thermosphere. One can see from Figure 5a that this behaviour is reproduced in the Collm data, although the trends are weaker and insignificant. Note that the negative wind trends in the lower layers cannot be seen in Figure 6a because this is masked by a strong solar cycle effect. During winter, the zonal wind trends are positive and decreasing with altitude as well. In both cases this means that the mesospheric wind jets have increased during the last 2-3 decades, but this effect decreases or even reverses with altitude.

Stronger mesospheric wind jets in the presence of saturation means larger $\mathrm{GW}$ amplitudes. Consequently, positive GW variance trends are expected in both summer 
and winter, which is indeed visible in Figure 4a. An exception is found in July, but one may assume that the zero trend line is simply shifted to an altitude just below $83 \mathrm{~km}$. The decreasing zonal mean wind trends lead to decreasing GW intrinsic phase speed trends and thus decreasing variance trends as well. During summer, the trends are mostly negative as expected from the positive prevailing wind trends. The decreasing or reversing zonal wind trends with altitude can be understood as an effect of GW momentum transfer. Stronger mesospheric jets lead to larger GW amplitudes, stronger wave drag and thus decreasing tendencies for the wind trends.

The solar cycle effect is stronger than the long term trend in both prevailing wind and $\mathrm{GW}$ variances. Figure $5 \mathrm{~b}$ shows that the effect is consistent with a stronger mesospheric jet during solar maximum both for summer and for winter. In both seasons the effect decreases with height and partly reverses. Note that the effect is strongest for early summer and early winter. For summer, this may be explained by the decrease of the zero wind line with time. In midwinter the wind field is dominated by stratospheric warmings and strong year-to-year variability, so that significant decadal effects are expected only in early winter.

Stronger mesospheric jets during solar maximum should, according to linear theory, be connected with larger $\mathrm{GW}$ variances in both summer and winter. This is indeed visible in Figure 4b. One may also note the decreasing GW solar cycle effect with altitude, which is due to the decreasing zonal wind trend with height.

\section{Conclusions}

It could be shown from 24 years of zonal wind and GW proxy analyses, that GW variances and mean winds exhibit trends which are qualitatively consistent with each other and may be explained by linear theory and a strengthening of the mesospheric wind jet during the last decades. Note, however, that the long-term trend coefficients presented are in most cases not significant, so that on the one hand the conclusions drawn must be considered as preliminary. On the other hand, however, the results for summer are consistent with medium frequency radar analyses by Hoffmann et al. (2011) so that the overall picture of long-term trends drawn here may be qualitatively correct.

During both summer and winter the mesospheric jets increase with solar activity. These increased jets are connected with increased GW amplitudes. Thus, as is the case with the long-term trends, solar cycle effects of GW may be explained by linear theory.

Still, however, the time series to be analysed are short. Long-term trends can only qualitatively be analysed from slightly more than 2 solar cycles of data. Unfortunately, the Collm LF time series have been terminated after 2007. Although the wind measurements are continued by meteor radar measurements (Jacobi, 2011) it is not yet clear whether trend analyses can be performed using these two datasets without the risk of artefacts leading to possible inhomogeneity of the combined time series. 


\section{Acknowledgements}

This study was supported by Deutsche Forschungsgemeinschaft under grant JA 836/22-1. F10.7 solar indices have been provided by NGDC through ftp://ftp.ngdc.noaa.gov/STP/SOLAR_DATA/.

\section{References}

Gavrilov, N. M., Jacobi, Ch., Kürschner, D., 2001: Climatology of ionospheric drift perturbations at Collm, Germany, Adv. Space Res., 27, 1779-1784.

Hoffmann, P., Rapp, M., Singer, W., Keuer, D., 2011: Trends of mesospheric gravity waves at northern middle latitudes during summer, J. Geophys. Res., 116, D00P08, doi:10.1029/2011JD015717.

Jacobi, Ch., Arras, C., Kürschner, D., Singer, W., Hoffmann, P., Keuer, D., 2009: Comparison of mesopause region meteor radar winds, medium frequency radar winds and low frequency drifts over Germany, Adv. Space. Res., 43, 247-252.

Jacobi, Ch., 2011: Meteor radar measurements of mean winds and tides over Collm $\left(51.3^{\circ} \mathrm{N}, 13^{\circ} \mathrm{E}\right)$ and comparison with LF drift measurements 2005-2007, Adv. Radio Sci. 9, 335-341.

Jacobi, Ch., Gavrilov, N.M., Kürschner, D., Fröhlich, K., 2006: Gravity wave climatology and trends in the mesosphere/lower thermosphere region deduced from lowfrequency drift measurements $1984-2003\left(52.1^{\circ} \mathrm{N}, 13.2^{\circ} \mathrm{E}\right)$, J. Atmos. Solar-Terr. Phys., 68, 1913-1923.

Kürschner, D., 1991: Ein Beitrag zur statistischen Analyse hochatmosphärischer Winddaten aus bodengebundenen Messungen, Z. Meteorol. 41, 1991, 262 - 266.

Kürschner, D., Schminder, R., Singer, W., Bremer, J., 1987: Ein neues Verfahren zur Realisierung absoluter Reflexionshöhenmessungen an Raumwellen amplitudenmodulierter Rundfunksender bei Schrägeinfall im Langwellenbereich als Hilfsmittel zur Ableitung von Windprofilen in der oberen Mesopausenregion, Z. Meteorol., 37, 322 - 332.

Schminder, R., Kürschner, D., 1988: Mean winds and tides in the 85- to 110-km region over Central Europe in 1983-1986, J. Geophys. Res., 93, 2493-2497.

Schminder, R., Kürschner, D., 1994: Permanent monitoring of the upper mesosphere and lower thermosphere wind fields (prevailing and semidiurnal tidal components) obtained from LF Dl measurements in 1991 at the Collm Geophysical Observatory, J. Atmos. Terr. Phys., 56, 1263-1269. 
Wissenschaftliche Mitteilungen aus dem Institut für Meteorologie der Universität Leipzig Bd. 50 